\title{
A representation formula for the inverse harmonic mean curvature flow
}

\author{
Knut Smoczyk
}

Knut Smoczyk promovierte im Jahr 1994 an der Ruhr-Universität Bochum. Danach folgten mehrere Forschungsaufenthalte in den USA, der Schweiz und in Deutschland. Seit 2000 ist er Privatdozent an der Universität Leipzig und seit April 2004 Heisenberg-Stipendiat am Albert-Einstein-Institut in Golm und am Max-Planck-Institut für Mathematik in den Naturwissenschaften in Leipzig.

\section{Introduction}

Let $M_{0}$ be a smooth, closed, strictly convex hypersurface in euclidean space $\mathbb{R}^{n+1}$ and suppose that $M_{0}$ is given by a smooth embedding $F_{0}: S^{n} \rightarrow \mathbb{R}^{n+1}$ of the unit $n$-sphere $S^{n}=\left\{x \in \mathbb{R}^{n+1}:|x|=1\right\}$. We consider the initial value problem for the inverse harmonic mean curvature flow

$$
\begin{gathered}
\frac{d}{d t} F(x, t)=\mathcal{H}^{-1}(x, t) v(x, t), \\
F(\cdot, 0)=F_{0},
\end{gathered}
$$

Im nachfolgenden Beitrag erhalten wir Einblick in einen aktuellen Forschungszweig der Differentialgeometrie. Bekanntlich lassen sich geometrische Strukturen auf Mannigfaltigkeiten mit besonderen Eigenschaften sehr oft durch partielle Differentialgleichungen beschreiben, eine Thematik, die auch bei den jüngsten Lösungsansätzen zur Poincaré Vermutung eine zentrale Rolle spielt. $\mathrm{Zu}$ diesen Gleichungen gehören beispielsweise die geometrischen Flussgleichungen, die im allgemeinen aus nichtlinearen Systemen parabolischer Differentialgleichungen bestehen. Die Lösbarkeit solcher Gleichungen bringt oft erhebliche Schwierigkeiten mit sich. So ist es in der Regel unmöglich, aus beliebigen Anfangsdaten die exakte Lösung zu einem späteren Zeitpunkt explizit zu berechnen. Umso erstaunlicher ist es, dass dies beim inversen harmonischen mittleren Krümmungsfluss dennoch möglich ist. Durch die Betrachtung einfacher Beispiele gelingt es dem Autor, den komplexen Gegenstand konkret zu illustrieren. 
where

$$
\mathcal{H}:=\frac{1}{\frac{1}{\kappa_{1}}+\cdots+\frac{1}{\kappa_{n}}}
$$

is the harmonic mean curvature of the hypersurface $M_{t}$ parameterized by $F_{t}:=F(\cdot, t)$ : $S^{n} \rightarrow \mathbb{R}^{n+1}, \kappa_{1}, \ldots, \kappa_{n}$ denote the principal curvatures of $M_{t}$ and $v(\cdot, t)$ is the outer unit normal vectorfield along $M_{t}$.

There are numerous important works on this flow. One should for example consult Andrews [3], [4], Chow-Liou-Tsai [8], Gerhardt [10] and Urbas [13]. It has been shown in Urbas [13] that $(*)$ admits a smooth solution for $t \in[0, \infty)$ and that the solutions tend to infinity as $t \rightarrow \infty$. Moreover, the hypersurfaces stay strictly convex and embedded and after a time dependent homothetic rescaling the rescaled hypersurfaces converge smoothly to a round sphere (see also Gerhardt [10] for an extension to starshaped hypersurfaces). In Chow-Liou-Tsai [8] the authors considered hypersurfaces driven by functions of the inverse harmonic mean curvature and also proved that convexity is preserved for a wide class of such flows, including $(*)$. Andrews [3], [4] treated both inward and outward directed flows.

For a geometric evolution equation it is in general not possible to determine the explicit solution. If $T$ denotes the first time where a singularity occurs, one rather studies the blowup behaviour of such flows as $t \rightarrow T$. Under certain conditions for the initial hypersurface it is often possible to classify the singularities, at least after a suitable rescaling procedure. E.g. under the assumption that the initial hypersurface is convex one was able to prove for a wide class of such flows (inward and outward directed) that a homothetically rescaled flow smoothly converges to a round sphere as $t \rightarrow T$.

If a convex hypersurface is evolving under the nonlinear parabolic equation $(*)$ given by the inverse harmonic mean curvature flow, it is therefore astonishing that it is possible to obtain the explicit solution. We state the main theorem:

Theorem 1.1. Let $M_{0}$ be a smooth, closed, strictly convex hypersurface in euclidean space $\mathbb{R}^{n+1}$ and suppose that $M_{0}$ is given by a smooth embedding $F_{0}: S^{n} \rightarrow \mathbb{R}^{n+1}$ of the unit $n$-sphere $S^{n}=\left\{x \in \mathbb{R}^{n+1}:|x|=1\right\}$. The inverse harmonic mean curvature flow

$$
\begin{gathered}
\frac{d}{d t} F(x, t)=\mathcal{H}^{-1}(x, t) v(x, t), \\
F(\cdot, 0)=F_{0},
\end{gathered}
$$

admits a smooth, strictly convex solution for $t \in[0, \infty)$.

The hypersurfaces $M_{t}:=F\left(S^{n}, t\right) \subset \mathbb{R}^{n+1}$ can be parameterized by their inverse Gauss maps $\mathcal{Y}_{t}: S^{n} \rightarrow M_{t}$ in the following way

$$
\mathcal{Y}_{t}(x)=D \bar{S}(x, t), \quad \text { for all }(x, t) \in S^{n} \times[0, \infty)
$$

where $\bar{S}(\cdot, t): \mathbb{R}^{n+1} \backslash\{0\} \rightarrow \mathbb{R}$ is the homogeneous extension of degree one of the support function $S(\cdot, t): S^{n} \rightarrow \mathbb{R}$ of $M_{t}$ defined by

$$
\bar{S}(\lambda x, t):=\lambda S(x, t), \quad \text { for all }(x, t) \in S^{n} \times[0, \infty) \text {, and all } \lambda>0 .
$$


Here, $D$ is the gradient in $\mathbb{R}^{n+1}$ and the support function $S(\cdot, t)$ is given by the formula

$$
S(x, t)=e^{n t} \int_{S^{n}} H(x, y, t) S(y, 0) d \sigma(y),
$$

where $H(x, y, t)$ is the heat kernel and $d \sigma$ the standard volume element on $S^{n} . S(\cdot, 0)$ denotes the support function of the initial hypersurface $M_{0}$.

Remark 1.2. The following theorem about the heat kernel is well-known (cf. BergerGauduchon-Mazet [5]):

Theorem. [5] Let $M$ be a compact Riemannian manifold, $\left\{f_{i}\right\}$ be an orthonormal basis of $L^{2}(M)$ consisting of eigenfunctions with corresponding eigenvalues $\lambda_{i}$ (i.e., $\Delta f_{i}=-\lambda_{i} f_{i}$ ), then

$$
H(x, y, t)=\sum e^{-\lambda_{i} t} f_{i}(x) f_{i}(y) .
$$

Moreover, the eigenfunctions $f_{k}$ on the unit $n$-sphere are the spherical harmonics $Y_{n, k}$ which are restrictions to $S^{n}$ of the homogeneous harmonic polynomials of degree $k$ in $\mathbb{R}^{n+1}$. They can be expressed in terms of the Legendre polynomials (see Müller [12] for more details on spherical harmonics).

Example 1.3. Let us briefly discuss the one-dimensional situation. If $n=1$, then $\mathcal{H}^{-1}=$ $\frac{1}{k}$, where $k$ denotes the curvature of the evolving convex curves $\gamma_{t}$. In this case, the flow

$$
\frac{d}{d t} \gamma_{t}=\frac{1}{k} v
$$

can also be viewed as the one-dimensional version of the inverse mean curvature flow

$$
\frac{d}{d t} F=\frac{1}{H} v
$$

which is important in General Relativity (see Huisken-Ilmanen [11] for details). The eigenvalues $\lambda_{k}$ of the Laplacian on $S^{1} \cong[0,2 \pi)$ are $\lambda_{k}=k^{2}, k \in \mathbb{N}$ with multiplicity 2 . Moreover, the functions $\frac{1}{\sqrt{\pi}} \cos (k x), \frac{1}{\sqrt{\pi}} \sin (k x)$ form an orthonormal basis of $L^{2}\left(S^{1}\right)$. For the heat kernel on $S^{1}$ we get

$$
H(x, y, t)=\frac{1}{\pi} \sum_{k \in \mathbb{N}} e^{-k^{2} t}(\cos (k x) \cos (k y)+\sin (k x) \sin (k y)) .
$$

According to Theorem 1.1, the support function $S(\cdot, t)$ of $\gamma_{t}$ is given by the formula

$$
S(x, t)=\sum_{k \in \mathbb{N}} e^{\left(1-k^{2}\right) t}\left(c_{k} \cos (k x)+s_{k} \sin (k x)\right),
$$

where the constants $c_{k}, s_{k}$ are defined by

$$
c_{k}:=\frac{1}{\pi} \int_{0}^{2 \pi} \cos (k y) S(y, 0) d y, \quad s_{k}:=\frac{1}{\pi} \int_{0}^{2 \pi} \sin (k y) S(y, 0) d y
$$


and $S(\cdot, 0)$ denotes the support function of the initial curve $\gamma_{0}$. If $\bar{S}$ denotes the extension of $S$ to $\mathbb{R}^{2} \backslash\{0\}$ as above, then

$$
D \bar{S}(x, t)=S(x, t)\left(\begin{array}{c}
\cos x \\
\sin x
\end{array}\right)+S^{\prime}(x, t)\left(\begin{array}{c}
-\sin x \\
\cos x
\end{array}\right), \quad \text { for all } x \in[0,2 \pi),
$$

where we have set

$$
S^{\prime}(x, t):=\frac{\partial}{\partial x} S(x, t)
$$

Consequently

$$
\begin{aligned}
\mathcal{Y}(x, t) & =\sum_{k \in \mathbb{N}} e^{\left(1-k^{2}\right) t} \cos (k x)\left(\begin{array}{l}
c_{k} \cos x-k s_{k} \sin x \\
c_{k} \sin x+k s_{k} \cos x
\end{array}\right) \\
& +\sum_{k \in \mathbb{N}} e^{\left(1-k^{2}\right) t} \sin (k x)\left(\begin{array}{l}
s_{k} \cos x+k c_{k} \sin x \\
s_{k} \sin x-k c_{k} \cos x
\end{array}\right)
\end{aligned}
$$

is the parameterization of $\gamma_{t}$ by the inverse Gauss map.

Example 1.4. We give an explicit example. Let $a \in[0,1)$ be a number and assume that the initial support function is given by

$$
S(y, 0)=1+a \sin ^{2}(y)=\frac{2+a}{2}-\frac{a}{2} \cos (2 y) .
$$

It then easily follows that

$$
\begin{gathered}
s_{k}=0 \text { for all } k \in \mathbb{N} \\
c_{0}=2+a, c_{2}=-\frac{a}{2} \text { and } c_{k}=0 \text { for all } k \in \mathbb{N} \backslash\{0,2\}
\end{gathered}
$$

By formula (1.2) the support function of the evolving curves $\gamma_{t}$ is

$$
S(x, t)=(2+a) e^{t}-\frac{a}{2} e^{-3 t} \cos (2 x)
$$

and the inverse Gauss maps are

$$
\mathcal{Y}(x, t)=\left((2+a) e^{t}-\frac{a}{2} e^{-3 t} \cos (2 x)\right)\left(\begin{array}{c}
\cos x \\
\sin x
\end{array}\right)+a e^{-3 t} \sin (2 x)\left(\begin{array}{c}
-\sin x \\
\cos x
\end{array}\right) .
$$

If we consider the rescaled curves $\tilde{\gamma}_{t}:=e^{-t} \gamma_{t}$, then the support functions $\tilde{S}$ and inverse Gauss maps $\tilde{\mathcal{Y}}(x, t)$ of $\tilde{\gamma}_{t}$ are

$$
\begin{gathered}
\tilde{S}(x, t)=2+a-\frac{a}{2} e^{-4 t} \cos (2 x), \\
\tilde{\mathcal{Y}}(x, t)=\left(2+a-\frac{a}{2} e^{-4 t} \cos (2 x)\right)\left(\begin{array}{c}
\cos x \\
\sin x
\end{array}\right)+a e^{-4 t} \sin (2 x)\left(\begin{array}{c}
-\sin x \\
\cos x
\end{array}\right) .
\end{gathered}
$$

In particular, if $t \rightarrow \infty$, then the support functions $\tilde{S}(x, t)$ tend to the constant $a+2$ which means that the curves converge uniformly to the circle of radius $a+2$ centered at the origin. Fig. 1 shows the flow for $a=-\frac{3}{4}$ at different time steps, Fig. 2 depicts the rescaled solution and Fig. 3 shows the curves in a single coordinate plane. 

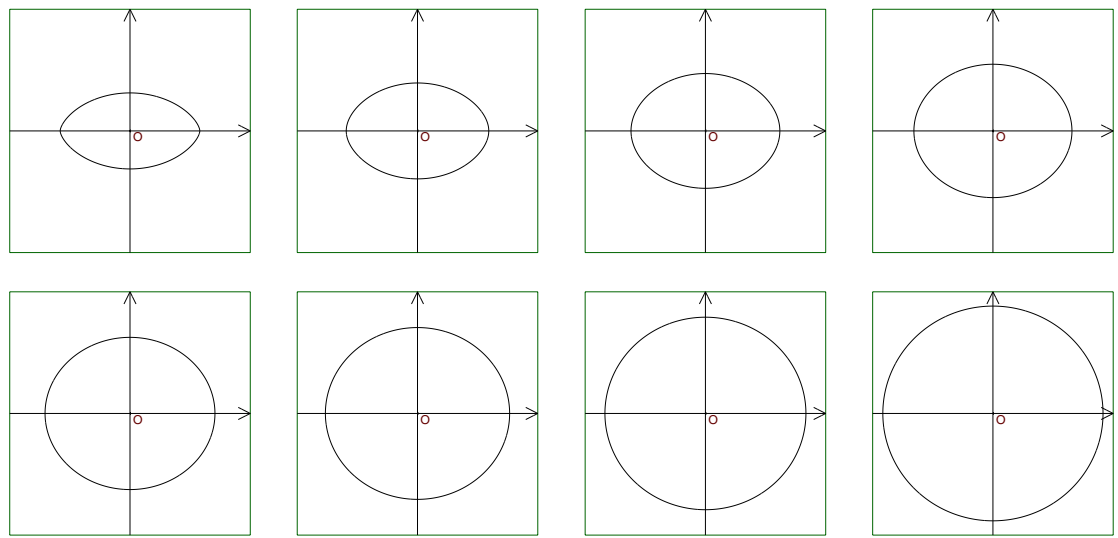

Fig. 1 The flow $\frac{d}{d t} \gamma_{t}=\frac{1}{k} v$ for the curve $\gamma_{0}$ with support function $S(x)=1-\frac{3}{4} \sin ^{2}(x)$ at the different time steps $t=\frac{j}{10}, j \in\{0,1,2,3,4,5,6,7\}$
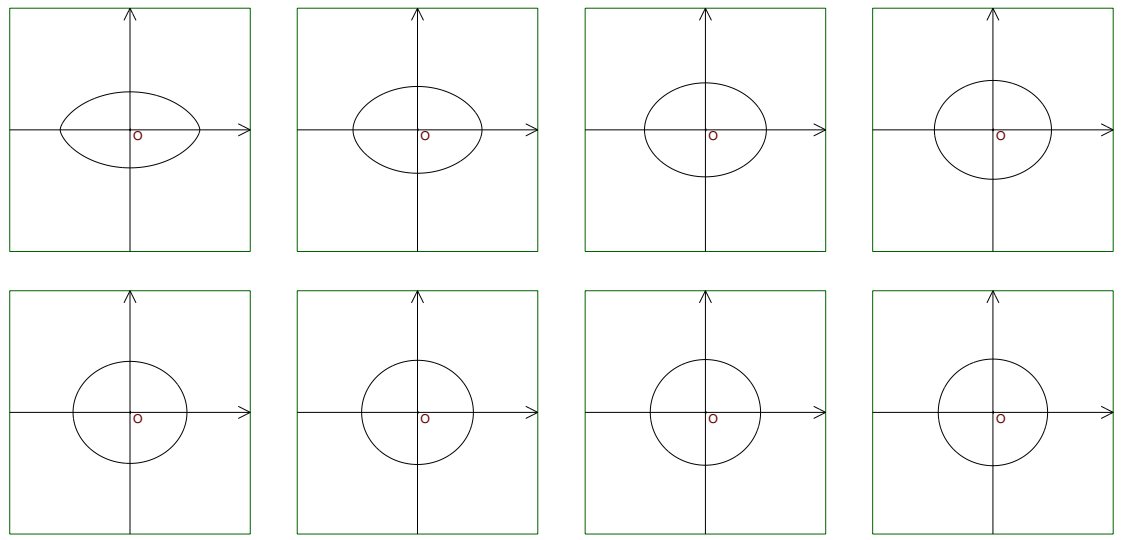

Fig. 2 The rescaled curves $\tilde{\gamma}_{t}=e^{-t} \gamma_{t}$ with $\gamma_{t}$ as in Fig. 1

\section{Support functions}

Let $M$ be a smooth, closed, strictly convex hypersurface in $\mathbb{R}^{n+1}$. We shall recall some facts about the support function of convex hypersurfaces (for more results see BonnesenFenchel [6]). Since $M$ is strictly convex, the Gauss map is invertible. Thus, we may assume that $M$ is parameterized by the inverse Gauss map $\mathcal{Y}: S^{n} \rightarrow M \subset \mathbb{R}^{n+1}$. This means that $v(x)=x$. Without loss of generality, we may assume that $M$ encloses the origin. The support function $S$ of $M$ is defined by

$$
S(x):=\langle x, \mathcal{Y}(x)\rangle \text { for all } x \in S^{n},
$$



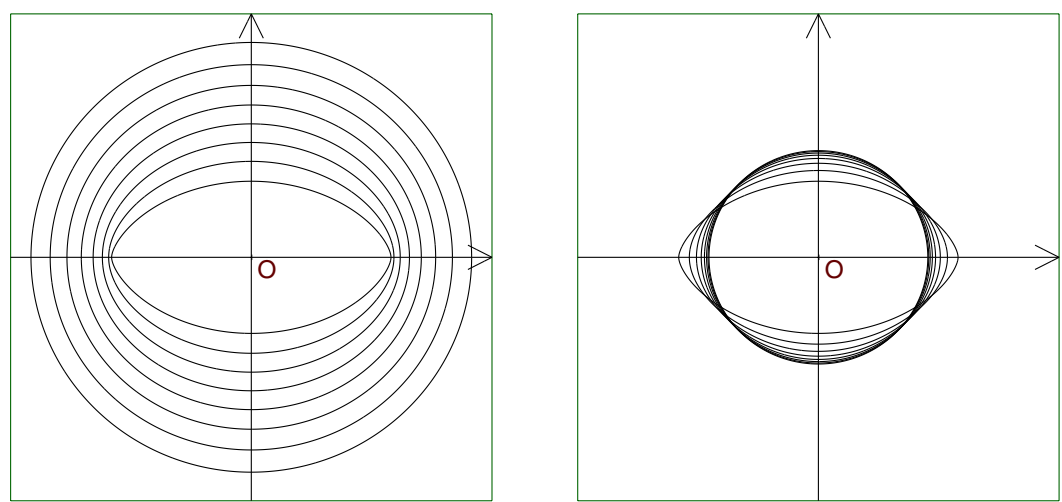

Fig. 3 The curves in Fig. 1 resp. Fig. 2 in a single coordinate plane

where $\langle\cdot, \cdot\rangle$ denotes the standard inner product of $\mathbb{R}^{n+1}$. One can extend $S$ to a homogeneous function $\bar{S}$ on $\mathbb{R}^{n+1} \backslash\{0\}$ of degree one by

$$
\bar{S}(\lambda x):=\lambda S(x) \text { for all } x \in S^{n} \text { and } \lambda>0 .
$$

It then follows

$$
D \bar{S}(x)=\mathcal{Y}(x) \text { for all } x \in S^{n},
$$

where $D \bar{S}$ is the gradient of $\bar{S}$ in $\mathbb{R}^{n+1}$. Let $\sigma=\sigma_{i j} d x^{i} \otimes d x^{j}$ denote the standard metric on $S^{n}$ and $\nabla$ its induced Levi-Civita connection. We want to compute the Hessian $\nabla^{2} S$ of $S$. We have

$$
\nabla_{i} S=\nabla_{i}\langle\mathcal{Y}, x\rangle=\left\langle\mathcal{Y}, \nabla_{i} x\right\rangle
$$

because $v(x)=x$ and $\left\langle\nabla_{i} \mathcal{Y}, v\right\rangle=0$. Taking another covariant derivative we obtain

$$
\nabla_{i} \nabla_{j} S=\left\langle\nabla_{i} \mathcal{Y}, \nabla_{j} x\right\rangle+\left\langle\mathcal{Y}, \nabla_{i} \nabla_{j} x\right\rangle
$$

The Gauss-Weingarten equations imply

$$
\nabla_{i} \nabla_{j} x=-\tau_{i j} x
$$

where $\tau_{i j}$ is the second fundamental form of $S^{n}$ and because $\tau_{i j}=\sigma_{i j}$ we have

$$
\nabla_{i} \nabla_{j} x=-\sigma_{i j} x
$$

On the other hand

$$
\left\langle\nabla_{i} \mathcal{Y}, \nabla_{i} x\right\rangle=\left\langle\nabla_{i} \mathcal{Y}, \nabla_{i} v\right\rangle=h_{i j}
$$

is the second fundamental form of $M$, so that we derive

$$
\nabla_{i} \nabla_{j} S=h_{i j}-\sigma_{i j} S .
$$


Moreover, the Weingarten equation gives

$$
\nabla_{i} v=h_{i j} g^{j k} \nabla_{k} \mathcal{Y}
$$

Then

$$
\sigma_{i j}=\left\langle\nabla_{i} x, \nabla_{j} x\right\rangle=\left\langle\nabla_{i} v, \nabla_{j} v\right\rangle=\left\langle h_{i k} g^{k l} \nabla_{l} \mathcal{Y}, h_{j s} g^{s t} \nabla_{t} \mathcal{Y}\right\rangle=h_{i k} h_{j l} g^{k l}
$$

so that

$$
\sigma_{i j}=h_{i k} h_{j l} g^{k l},
$$

where $g^{k l}$ is the inverse of the induced metric $g_{i j}$ on $M$. From (2.1) and (2.2) we immediately obtain

$$
\Delta S=\sigma^{i j} \nabla_{i} \nabla_{j} S=\mathcal{H}^{-1}-n S .
$$

Next we will compute the evolution equation of the support function $S$. To this end let us assume that $M_{t}$ is a smooth family of closed, strictly convex hypersurfaces in $\mathbb{R}^{n+1}$ parameterized by a smooth embedding $F_{t}: S^{n} \rightarrow M_{t} \subset \mathbb{R}^{n+1}$ such that

$$
\frac{d}{d t} F_{t}(x)=f(x, t) v(x, t)
$$

where $f(x, t)$ is a smooth speed function. It is then possible to find a uniquely determined diffeomorphism $\Psi_{t}: S^{n} \rightarrow S^{n}$ such that the embedding

$$
\mathcal{Y}_{t}: S^{n} \rightarrow M_{t}, \quad \mathcal{Y}_{t}(x):=F_{t}\left(\Psi_{t}(x)\right)
$$

is the inverse Gauss map. Thus, we obtain

$$
\begin{aligned}
\frac{d}{d t} S_{t} & =\frac{d}{d t}\left\langle\mathcal{Y}_{t}(x), x\right\rangle=\frac{d}{d t}\left\langle F_{t}\left(\Psi_{t}(x)\right), x\right\rangle \\
& =\left\langle\frac{\partial}{\partial t} F_{t}\left(\Psi_{t}(x)\right)+D F_{t}\left(\frac{\partial \Psi}{\partial t}\right), x\right\rangle=\left\langle\frac{\partial}{\partial t} F_{t}\left(\Psi_{t}(x)\right), x\right\rangle \\
& =\left\langle f\left(\Psi_{t}(x), t\right) v\left(\Psi_{t}(x), t\right), x\right\rangle=f .
\end{aligned}
$$

In particular, if $f$ is given by the inverse of the harmonic mean curvature, then (2.3) implies

Lemma 2.1. If $M_{t}$ is a smooth family of closed, strictly convex hypersurfaces in $\mathbb{R}^{n+1}$ evolving by the inverse harmonic mean curvature flow $(*)$, then the support function satisfies the linear equation

$$
\frac{d}{d t} S=\Delta S+n S
$$

where $\Delta$ is the Laplacian w.r.t. the standard metric on $S^{n}$.

Corollary 2.2. If $M_{t}$ is a smooth family of closed, strictly convex hypersurfaces in $\mathbb{R}^{n+1}$ evolving by the inverse harmonic mean curvature flow $(*)$, then the support function $S(\cdot, t)$ of $M_{t}$ is given by

$$
S(x, t)=e^{n t} \int_{S^{n}} H(x, y, t) S(y, 0) d \sigma(y),
$$

where $H(x, y, t)$ is the heat kernel on $S^{n}$ and $d \sigma$ the standard volume element on $S^{n}$. 
Proof. The function $\tilde{S}(x, t):=e^{-n t} S(x, t)$ satisfies the heat equation

$$
\frac{d}{d t} \tilde{S}=\Delta \tilde{S}
$$

and then

$$
\tilde{S}(x, t)=\int_{S^{n}} H(x, y, t) \tilde{S}(y, 0) d \sigma(y) .
$$

But since $\tilde{S}(y, 0)=S(y, 0)$ we obtain the result.

Corollary 2.3. Let $M_{0}$ be a smooth, closed, strictly convex hypersurface in $\mathbb{R}^{n+1}$ and let $M_{t}$ be the corresponding smooth family of hypersurfaces evolving by their inverse harmonic mean curvature. Then the rescaled hypersurfaces $\tilde{M}_{t}:=e^{-n t} M_{t}$ converge smoothly to a round sphere centered at the origin as $t \rightarrow \infty$.

Proof. If $S(\cdot, t)$ and $\tilde{S}(\cdot, t)$ are the support functions of $M_{t}$ resp. $\tilde{M}_{t}$, then

$$
\tilde{S}(x, t)=e^{-n t} S(x, t) .
$$

In addition, by equation (2.4) $\tilde{S}$ solves the heat equation on $S^{n}$ and therefore smoothly converges to a constant as $t \rightarrow \infty$. It is clear that a smooth convergence of the support function implies a smooth convergence of the corresponding hypersurfaces as well. On the other hand, the support function is constant if and only if the hypersurface is a round sphere centered at the origin.

Proof of the main theorem. It is well-known that a solution of $(*)$ exists for $t \in[0, \infty)$ and that the hypersurfaces $M_{t}$ stay convex and embedded during the flow (cf. Urbas [13]). It is also well-known that the rescaled hypersurfaces $\tilde{M}_{t}:=e^{-n t} M_{t}$ converge smoothly to a round sphere centered at the origin. It remains to prove the precise formula for the support function and the inverse of the Gauss maps. This has been shown in Corollary 2.2 and the equation for the inverse of the Gauss maps $\mathcal{Y}$ follows from $\left.D \bar{S}\right|_{S^{n}}=\mathcal{Y}$.

\section{Acknowledgements}

The work presented in the first section was completed while the author stayed at the Max Planck Institute for Mathematics in the Sciences in Leipzig. He wants to express his gratitude to Jürgen Jost for his support.

\section{References}

[1] Anada, K.: Contraction of surfaces by harmonic mean curvature flows and nonuniqueness of their self similar solutions. Calc. Var. Partial Differential Equations 12 (2001), 109-116.

[2] Anada, K.; Tsutsumi, M.: Stability of solutions of nonlinear parabolic equations for harmonic mean curvature flows. Nonlinear Anal. 51 (2002), 305-319.

[3] Andrews, B.: Evolving convex hypersurfaces. Martin, G. et al. (eds.), Proceedings of the miniconference on analysis and applications, held at the University of Queensland, Brisbane, Australia, September 2023, 1993. Canberra: Australian National University, Centre for Mathematics and its Applications. Proc. Centre Math. Appl. Austral. Nat. Univ. 33 (1994), 1-24. 
[4] Andrews, B.: Contraction of convex hypersurfaces in Riemannian spaces. J. Differential Geom. 39 (1994), $407-431$.

[5] Berger, M.; Gauduchon, P.; Mazet, E.: Le spectre d'une variété riemannienne. Lecture Notes in Math. 194, Springer-Verlag, 1971.

[6] Bonnesen, T.; Fenchel, W.: Theorie der konvexen Körper. Springer-Verlag, 1934.

[7] Chavel, I.: Eigenvalues in Riemannian geometry. Including a chapter by Burton Randol. With an appendix by Jozef Dodziuk. Pure Appl. Math. 115, Academic Press, Inc., Orlando, FL, 1984.

[8] Chow, B.; Liou, L.-P.; Tsai, D.-H.: On the nonlinear parabolic equation $\partial_{t} u=F(\Delta u+n u)$ on $S^{n}$. Comm. Anal. Geom. 4 (1996), 415-434.

[9] Chow, B.; Tsai, D.-H.: Expansion of convex hypersurfaces by nonhomogeneous functions of curvature. Asian J. Math. 1 (1997), 769-784.

[10] Gerhardt, C.: Flow of nonconvex hypersurfaces into spheres. J. Differential Geom. 32 (1990), $299-314$.

[11] Huisken, G.; Ilmanen, T.: The inverse mean curvature flow and the Riemannian Penrose inequality. $J$. Differential Geom. 59 (2001), 353-437.

[12] Müller, C.: Spherical harmonics. Lecture Notes in Math. 17, Springer-Verlag, 1966.

[13] Urbas, J.I.E.: An expansion of convex hypersurfaces. J. Differential Geom. 33 (1991), 91-125; Correction to, ibid. 35 (1992), 763-765.

Knut Smoczyk

Max Planck Institute for Mathematics in the Sciences

Inselstr. 22-26

D-04103 Leipzig, Germany

e-mail: Knut.Smoczyk@mis.mpg.de 